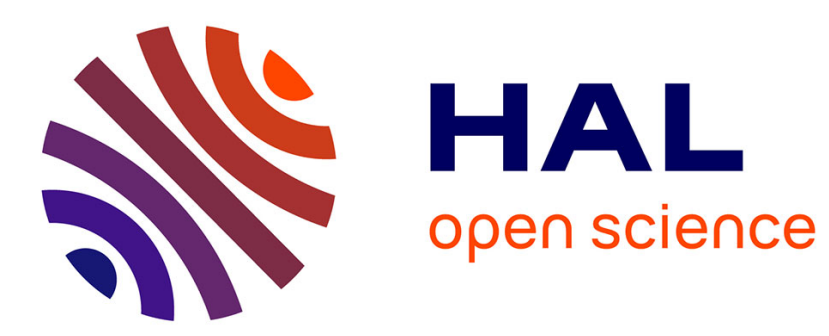

\title{
Experiments on helical vortex pairs in the wake of a rotor
}

Dominic Schröder, Ralf Hörnschemeyer, Eike Stumpf, Thomas Leweke

\section{To cite this version:}

Dominic Schröder, Ralf Hörnschemeyer, Eike Stumpf, Thomas Leweke. Experiments on helical vortex pairs in the wake of a rotor. AIAA Scitech 2021 Forum, Jan 2021, VIRTUAL EVENT, United States. 10.2514/6.2021-1088 . hal-03416209

\section{HAL Id: hal-03416209 \\ https://hal.science/hal-03416209}

Submitted on 5 Nov 2021

HAL is a multi-disciplinary open access archive for the deposit and dissemination of scientific research documents, whether they are published or not. The documents may come from teaching and research institutions in France or abroad, or from public or private research centers.
L'archive ouverte pluridisciplinaire HAL, est destinée au dépôt et à la diffusion de documents scientifiques de niveau recherche, publiés ou non, émanant des établissements d'enseignement et de recherche français ou étrangers, des laboratoires publics ou privés. 


\title{
Experiments on helical vortex pairs in the wake of a rotor
}

\author{
D. Schröder*, R. Hörnschemeyer ${ }^{\dagger}$ and E. Stumpf ${ }^{\ddagger}$ \\ RWTH Aachen University, 52062 Aachen, Germany \\ T. Leweke ${ }^{\S}$ \\ CNRS, Aix-Marseille Université, Centrale Marseille, 13384 Marseille, France
}

\begin{abstract}
This paper presents the results of an experimental study of the dynamics of two closely spaced helical vortices, generated in a water channel by a one-bladed rotor equipped with a perpendicular tip fin placed on its pressure side. The objective of this modification of the blade geometry is to produce a final tip vortex with increased core size and reduced vorticity and swirl velocities, by taking advantage of the mutual interactions and merging of the two initial vortices. The merged vortex is expected to lead to less severe interactions with a following blade, which cause vibrations and noise in certain regimes of rotor operation (blade-vortex interactions). The rotor wake is analyzed in a configuration close to hover through qualitative dye visualizations and quantitative measurements using stereoscopic particle image velocimetry. The modified blade geometry is found to produce a pair of concentrated tip vortices, whose circulation ratio and separation distance depend on the fin position and dimensions. Rapid growth of the vortex core radii prior to the onset of vortex merging indicates the presence of an instability phenomenon, which is here identified as a centrifugal-type instability. For circulation ratios close to 1 , the final vortex has a significantly larger core size than the single tip vortex of the unmodified blade. For lower values of this ratio, the merging is only partial and the increase of core size less pronounced. First results concerning counter-rotating tip vortices are also presented.
\end{abstract}
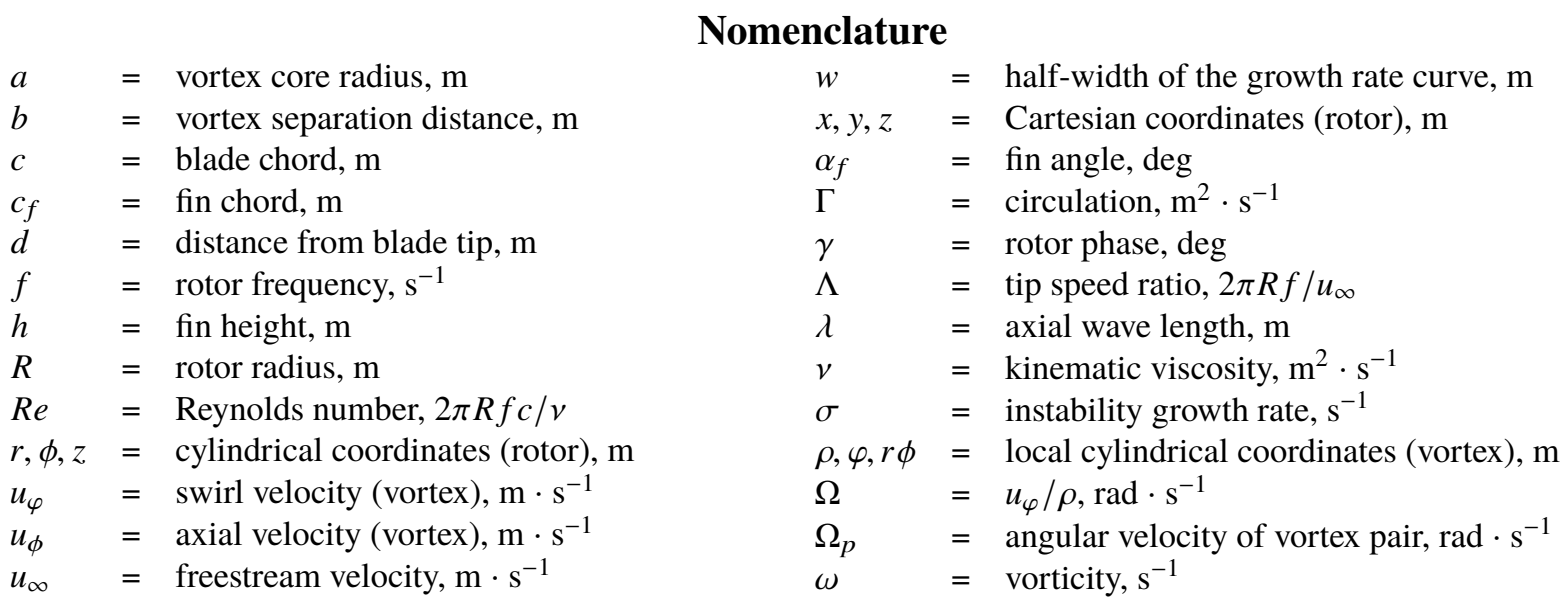

\section{Introduction}

Engineering applications involving rotors are characterized by the presence of helical vortices in the rotor wakes. Rotating blades, similar to fixed wings, cause the formation of concentrated vortices at their tips, as a consequence of the generated lift. Due to the combination of free-stream velocity and rotational motion of the blades, the vortices form a helical geometry while being convected downstream. These helical vortex wakes and their dynamics are relevant for many practical issues actively investigated in aerodynamic research today. With regard to helicopters, the helical tip vortices and their interaction with a following blade (Blade-Vortex-Interaction, BVI) can generate unwelcome noise in certain flight regimes and provoke unwanted vibrations of the structure [1]. For wind turbines, the interaction of the blades with the concentrated tip vortices in the wake of another wind turbine placed upstream can affect performance and fatigue due to unsteady loads caused by this interaction [2]. For both phenomena, the circulation and the core radius

\footnotetext{
*Researcher and PhD Student, Institute of Aerospace Systems (ILR)

${ }^{\dagger}$ Academic Counselor, Institute of Aerospace Systems (ILR)

$\doteqdot$ Professor, Head of Institute, Institute of Aerospace Systems (ILR) 1

${ }^{\S}$ Senior Researcher, Institut de Recherche sur les Phénomènes Hors Équilibre (IRPHE)
} 
of the vortices are crucial parameters affecting the strength of the interactions [3]. Reducing the negative effects of BVI has been the topic of numerous studies, resulting in a variety of concepts for rotor control. The current approaches can be divided into two groups, involving active and passive systems. A broad scope of active-control applications is presented in the review by Yung et al. [4]. The governing concept for passive systems is to influence the relevant vortex parameters, in order to decrease the negative effects resulting from the interaction with a following blade. Various concepts for blade tip geometries and their influence on the vortices have been investigated, most of them aiming at splitting or diffusing the tip vortex [5]. One operating principle for such tip shapes is based on creating multiple vortices, with their interactions resulting in a modification of the final vortex encountering the following blade. The stability of both the entire rotor wake and the single helical vortex filament is of significant importance regarding possible BVI reduction mechanisms. A detailed analysis leading to a deeper physical understanding of the interaction phenomena occurring in such closely spaced helical vortex systems is the objective of the ongoing French-German research project TWIN-HELIX. By means of a special blade tip geometry, consisting of a parametric fin mounted on the pressure side near the tip of a rotating blade, the concentrated tip vortex is split up into two separate vortices, which interact with each other while being propagated downstream. Variation of the geometric parameters have an effect on the properties of the initial vortex pair emerging from the blade, which will eventually merge into a single vortex. The time scale and outcome of the merging process is strongly affected by the characteristics of the initial vortex system, which may be receptive to various interaction and instability mechanisms. The objective here is to identify configurations leading to the generation of a merged vortex exhibiting an increased core size and a reduced vorticity peak, and that therefore offer a potential to reduce the negative effects associated with BVI and other vortex-structure interactions.

\section{Theoretical Background}

Vortex systems consisting of two like-signed (co-rotating) vortices eventually merge into one single vortex in a viscous flow. This phenomenon and the underlying physics have been studied extensively in the past, with regard to the decay of two-dimensional turbulence and the formation of larger structures. Merging involves a significant change in the vortex characteristics, between the initial two vortices and the final merged vortex. The vortex core radius $a_{\max }$, defined as distance between the vortex center and the point of maximum swirl velocity (see Fig. 3, is increased, whereas the peak vorticity in the core region is decreased [6]. The two-dimensional dynamics of the merging process is described in detail by Leweke et al. [7]. In this framework, two co-rotating vortices of circulations $\Gamma_{1}$ and $\Gamma_{2}$, whose centers are separated by a distance $b_{0}$, rotate around each other with an angular velocity $\Omega_{p}=\left(\Gamma_{1}+\Gamma_{2}\right) /\left(2 \pi b_{0}^{2}\right)$. The relevant parameter determining the onset of the merging process is the ratio between core radius and separation distance. Whereas the separation distance remains practically constant [8], the core size increases in time due to viscous diffusion of vorticity. If one defines the equivalent Gaussian core radius as $a \approx a_{\max } / 1.12$, previous studies have shown that merging sets in when the ratio $a / b_{0}$ exceeds a critical value of approximately 0.24 [7]. The initial diffusive stage is then followed by a convective phase, where the vorticity is rapidly reorganised, a second diffusive stage, and finally the merged diffusive stage. This sequence is descried in detail by Melander et al. [9] and Cerretelli \& Williamson [8]. The outcome of the merging process is affected by the parameter values of the initial vortex pair. For two-dimensional systems, Meunier et al. [6] showed that the final core radius is proportional to the initial vortex distance $b_{0}$ and exhibits maximum values for circulation ratios $\Gamma_{1} / \Gamma_{2} \approx 1$. Based on these findings, the objective for the present study with a modified blade geometry is therefore the generation of two vortices of nearly equal strength.

In addition to the final vortex parameters, the time scale of merging is also important for the planned application, since the entire process has to be completed before the encounter with a following rotor blade. The time before the onset of merging depends on the initial vortex pair parameters. In this first stage, the ratio $a / b_{0}$ is mainly influenced by the growth of the core radii, since the separation distance remains contant. In unperturbed systems, the core radii grow primarily due to viscous diffusion according to

$$
a=\sqrt{a_{0}^{2}+4 v t}
$$

where $a_{0}$ is the initial core radius (at time $t=0$ ) and $v$ the kinematic viscosity of the fluid. For high Reynolds numbers, this growth is rather slow. However, mutual interactions between the vortices might favor instability phenomena, which accelerate the growth and eventually the merging process. Similarly to straight vortices, Leweke et al. [10] divide the instability mechanisms for helical vortices into two groups, according to the resulting effects. One mechanism causes displacements of the whole vortex, with minimal effects on the internal core structure. Considering the associated wavelengths of the perturbations, in comparison to the vortex core diameter, these interactions are described as 
long-wavelength instabilities. Widnall [11] investigated the stability of a single helical vortex filament. A theoretical analysis of multiple interlaced helical vortices was performed by Gupta \& Loewy [12]. Analytical solutions for the velocity field induced by a helical vortex filament were provided by Kawada [13] and Hardin [14]. This work was extended by Okulov [15], providing an analytical solution for the generalized stability problem for multiple helical vortices. Experimental studies focussing on the long-wave instability of helical vortices were presented by Quaranta et al. [16, 17]. A second type of instability arises inside the vortex cores, with perturbation wavelengths of the order of the core diameter. Moore \& Saffman [18] identified the resonance of Kelvin modes (neutral linear perturbation modes of an axisymmetric vortex) with an imposed strain field as the origin of this instability. Kerswell [19] described this phenomenon with the term used today, the elliptic instability. Experimental evidence of its existence in straight vortex pairs was given by Leweke \& Williamson [20] and Meunier \& Leweke [21]. A numerical study investigating the influence of axial core flow on the elliptic instability was presented by Roy et al. [22], followed by an experimental study [23] of short-wave instabilities in both co- and counter-rotating vortex pairs with axial flow. Blanco-Rodríguez \& Le Dizès [24] listed curvature and torsion as other sources of short-wave instabilities responsible for internal core deformations in helical vortex systems. They provided an analytical proof that curvature adds a contribution to the elliptic instability growth rate [25]. Experimental evidence regarding short-wavelength instabilities in helical vortices can be found in [10]. Besides the elliptic instability, another short-wavelength phenomenon occurs in vortices exhibiting a curved axis, the curvature instability. Fukumoto \& Hattori [26] investigated the curvature instability of a vortex ring and pursued their work for helical vortex systems [27, 28]. Blanco-Rodríguez \& Le Dizès [29] presented an analytical investigation of the curvature instability in a helical vortex using the Batchelor model. A comparison between both short-wavelength instability mechanisms was provided by Hattori et al. [30], identifying the vortex swirl (ratio of swirl and axial velocities in the core) as an essential parameter. In addition to these mutual-interaction instabilities, which develop in the presence of a secondary vortex, rotational flows can also be affected by another form of instability. The centrifugal instability, first described by Lord Rayleigh [31], arises in vortices when the absolute value of the circulation decreases with increasing distance from the vortex center, i.e. when there is a region of opposite-signed vorticity surrounding the vortex core [32]. In such a region, the flow is unstable with respect to three-dimensional perturbations. Studies of the centrifugal instability and its physical origin were provided by Bayly [33] and Billant \& Gallaire [32, 34].

Vortex merging is significantly influenced by the presence of instabilities. Due to their three-dimensional characteristics, the instabilities transform the merging process into a more complex phenomenon. Meunier et al. [21, 35] pointed out that the criterion for onset and the characteristic parameters of the final merged vortex are strongly affected, compared to unperturbed systems. The critical ratio $a / b_{0}$ for the onset of three-dimensional merging was found to be smaller than the corresponding value for the two-dimensional case. In addition, instability influence leads to a larger core size. Meunier et al. [21] found an increase by a factor 3.5 for the merging of straight co-rotating vortices under the influence of elliptic instability, compared to a factor of 1.5 for the unperturbed case. The development of three-dimensional instabilities prior to merging therefore appears to be beneficial for the objective of the present study. Their evolution also depends on the initial vortex pair parameters. In particular, the growth rate of both long- and shortwave instabilities is proportional to $b_{0}^{-2}$. A rapid evolution would therefore require a small $b_{0}$, which is opposite to the requirement for a large radius of the merged vortex, suggesting the existence of an optimum value. Finding this optimum for the case of a rotating blade is one of the goals of the present study.

In this contribution, we show results from a series of experiments conducted in water, using a single-bladed rotor equipped with a fin to generate a pair of tip vortices of comparable circulation. The fin geometry and position are varied to obtain different initial vortex pair parameters and study the effect on interaction phenomena, such as instabilities and merging, and to perform an optimization with respect to the merging time scales and final vortex parameters.

\section{Experiments}

The present experimental study was carried out in the large recirculating water channel of the Institute of Aerospace Systems, having a test section of dimensions $1.5 \mathrm{~m}$ (width) $\times 1 \mathrm{~m}$ (height) $\times 6.5 \mathrm{~m}$ (length). A simplified one-bladed rotor is used to analyze the dynamics of a helical tip vortex pair. The blade cross section is a NACA 0012 profile, and its span (rotor radius) is $R=240 \mathrm{~mm}$, with a constant chord $c=80 \mathrm{~mm}$ in the outer $75 \%$ of the span. A slight twist is added to the geometry, for the rotor to operate in conditions close to hover. To ensure a stationary flow around the rotor, a small free-stream velocity $u_{\infty}$ in the water channel is needed to prevent the flow from recirculating around the rotor disk. The nominal rotation rate is $1 \mathrm{~Hz}$, resulting in a Reynolds number, based on the blade chord and tip velocity, of $R e=2 \pi R f c / v=120,000$. The simple rectangular blade geometry was chosen in order to focus on the basic mechanisms of generating two tip vortices. The relatively large chord length is justified by the need to have a 


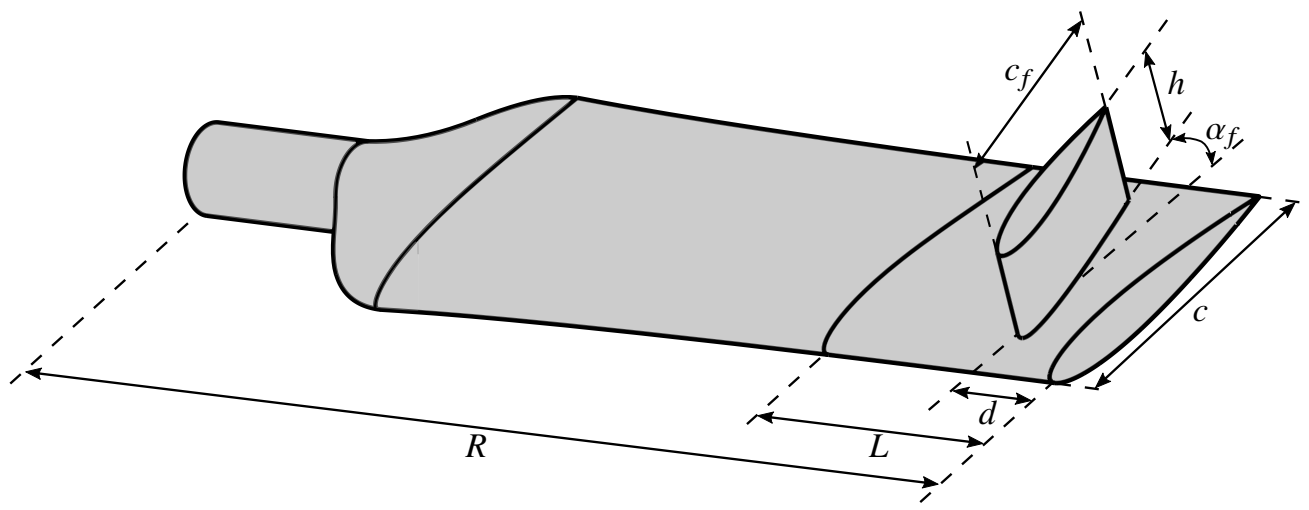

Fig. 1 Schematic of the blade and fin geometries. The blade tip, of span $L=60 \mathrm{~mm}$, is manufactured by $3 \mathrm{D}$ printing and interchangeable, to enable a variety of parameter combinations.

sufficiently large core size of the tip vortex for resolved particle image velocity (PIV) measurements in the core region. In order to generate a second tip vortex, a perpendicular fin is located near the tip on the suction side of the blade. Like the blade, the fin has a NACA 0012 cross section and a rounded tip. The parameters defining the fin geometry, i.e. its position $d$, height $h$, chord $c_{f}$ and angle of attack $\alpha_{f}$, can be varied, in order to explore different configurations of the tip vortex system, which are characterized by the ratio of vortex circulations and the distance between the vortex centers. The geometry of the rotor blade and the geometric parameters of the fin are shown in Fig. 11 For each parameter combination, a separate interchangeable tip geometry was manufactured by rapid prototyping (3D printing).

Two preliminary studies had been carried out, in order to establish the feasibility of this approach to generate helical vortex pairs. In the first, the configuration of a fixed (non-rotating) rectangular wing having the same NACA 0012 profile was used for an extensive parameter study concerning the fin geometry. This showed that the generation of a second tip vortex is much more robust when the fin is placed on the pressure side of the wing, and the parameters for a circulation ratio close to 1 were determined. In the second preliminary study, a small-scale rotor $1 / 3$ the scale of the one described above was tested in a $38 \mathrm{~cm} \times 45 \mathrm{~cm} \times 150 \mathrm{~cm}$ test section water channel, in order to verify the validity of the approach for a rotating wing. The geometry of the rotor was determined using a model of trailing helical vortex filaments, aiming to reproduce a similar spanwise circulation distribution near the tip as found for the fixed wing, in hover conditions. This investigation confirmed that the low aspect ratio of the blade, chosen in order to have sufficient spatial resolution for PIV measurements in the tip vortex cores, does not prevent the formation of concentrated helical vortices, and that the fin located on the pressure side operates in a similar way as for the fixed wing.

The rotor is mounted on a shaft having a length of 1.8 rotor diameters, driven through a gear box by a motor outside the test section. Linear axes enable an adaptable positioning of the rotor in the test section with high accuracy. Dye visualization of the rotor wake is achieved using fluorescein activated by ultraviolet light, providing information about the spatial evolution of the initial vortex system. Two internal channels in both the blade and the tip were used to inject the dye solution into the flow. The rotor was designed with a hollow shaft to allow the dye feed through internal tubes. A controllable linear axis was used to adjust the volumetric flow rate to the rotational speed of the rotor. Image acquisition was made at a frame rate of $300 \mathrm{~Hz}$ using a 12-megapixel XIMEA CB120xG-CM camera.

The properties of the tip vortex system were determined from velocity fields in the symmetry plane of the rotor. These fields were obtained in the water channel by Stereoscopic Particle Image Velocimetry (SPIV), which gives access to the three velocity components of the fluid in a two-dimensional field of view. PIV images were recorded by two CCD cameras (PCO.2000) with a resolution of $2048 \times 2048$ pixels. Both cameras had a collective angle of approximately $30^{\circ}$, following the recommendation of Lawson \& $\mathrm{Wu}[36]$ to minimize measurement errors in both the in-plane and out-of-plane velocity reconstruction. Water-filled prisms were used to prevent aberrations due to the change of fluids in the optical path, as suggested by Prasad \& Jensen [37] for optical measurement techniques in water channels. To ensure a uniformly focussed image, adapters fulfilling the Scheimpflug criterion were mounted in front of the cameras. The flow was illuminated by a double-pulsed laser (Quantel Twins Ultra) providing a pulse energy of $120 \mathrm{~mJ}$ at an effective wavelength of $532 \mathrm{~nm}$. Cubic polyamide particles of mean diameter $50 \mu \mathrm{m}$ and density $1.016 \mathrm{~g} / \mathrm{cm}^{3}$ were used to seed the flow. To prevent peak-locking errors, the foci were adjusted to secure a mean particle diameter of $2 \mathrm{~mm}$ in the recorded images [38]. An optical encoder combined with micro-controller was used to ensure a synchronized triggering of camera and laser at the desired rotor phase $\gamma$. Both the entire rotor wake and a close-up field of view 
a)

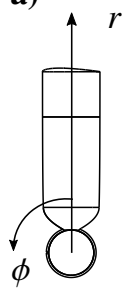

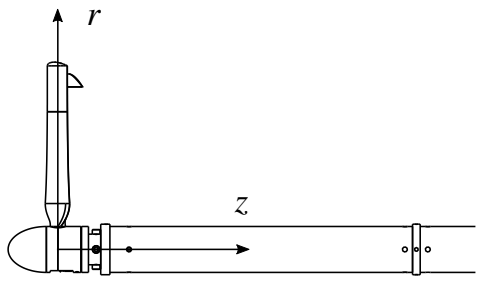

b)

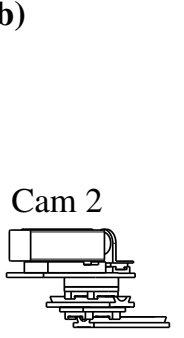

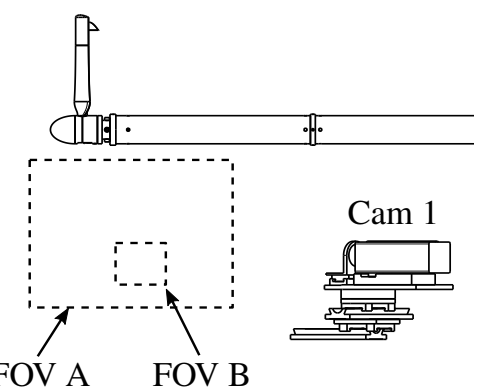

Fig. 2 a) Rotor geometry and coordinate system. The free-stream velocity is in the $z$-direction. b) Camera set-up and fields of view (FOV) for the PIV measurements.
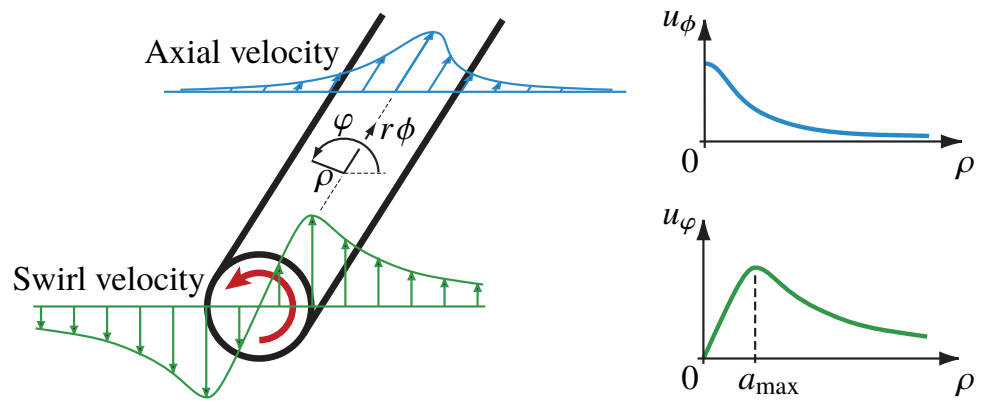

Fig. 3 Azimuthal $(\varphi)$ and axial $(\phi)$ velocity profiles characterizing a vortex, illustration adapted from [16].

containing the vortex interaction with high spatial resolution were investigated with Stereo-PIV. A wide field of view (FOV A), mapping a $300 \mathrm{~mm} \times 220 \mathrm{~mm}$ region of the flow was used to evaluate the entire wake and the spatial evolution of the vortex system. Using the information from the dye visualizations, a second field of view (FOV B) with an edge length of $80 \mathrm{~mm}$ was recorded to obtain a higher spatial resolution in the relevant flow regions containing the vortex cores. In Fig. 2, the coordinate system and the position of both fields of view relative to the rotor are shown. The light sheet was positioned directly below the axis of the rotor shaft. For FOV B, the rotor was adjusted in its $z$ - and $r$-position using linear axes, so that the vortices were always well-centred in the frame. The wake was recorded in $18^{\circ}$-steps of the rotor angle $\gamma$ to give insight into the temporal and spatial evolution. For each phase, 250 frames using the double-frame/single-pulse technique were captured. For calibration, image acquisition, synchronization of light source and cameras, as well as for image processing, the DaVis 8.4 software developed by LaVision was used.

A concentrated vortex is characterised by its radial profiles of azimuthal (swirl) and axial velocities, as illustrated schematically in Fig. 3, where the corresponding local cylindrical reference frame $(\rho, \varphi, r \phi)$ is also shown. The radial distance $a_{\max }$ from the vortex center where the swirl velocity is maximum is a measure of the vortex core size. The strength of the vortex is given by the value of its circulation $\Gamma$. $\Gamma$ and $a_{\max }$ will be used in the following to describe and compare the vortices in the rotor wake.

\section{Results}

We now present the experimental results and the corresponding analysis concerning the effect of a tip fin on the development of the wake vortex system of the rotor used in this study. For all cases discussed below, the rotation rate is $f=1 \mathrm{~Hz}$ and the tip Reynolds number $R e=120,000$. The free-stream velocity is $u_{\infty}=11.4 \mathrm{~cm} / \mathrm{s}$, resulting in a tip speed ratio $\Lambda=2 \pi R f / u_{\infty}=13$. The fin height and chord are also constant, at $h=24 \mathrm{~mm}=0.3 c$ and $c_{f}=64 \mathrm{~mm}=0.8 c$, respectively, whereas the fin position $d$ and angle of attack $\alpha_{f}$ is varied. We start by showing a comparison between the unmodified blade and a case with a fin leading to a significant change of the tip vortex properties, followed by an analysis of the instability mechanism involved in this process. We then discuss the effect of varying the geometrical fin parameters and identify two qualitatively different scenarios for the merging of the two co-rotating tip vortices. Some preliminary results concerning configurations where the fin creates a counter-rotating secondary vortex are also shown. 


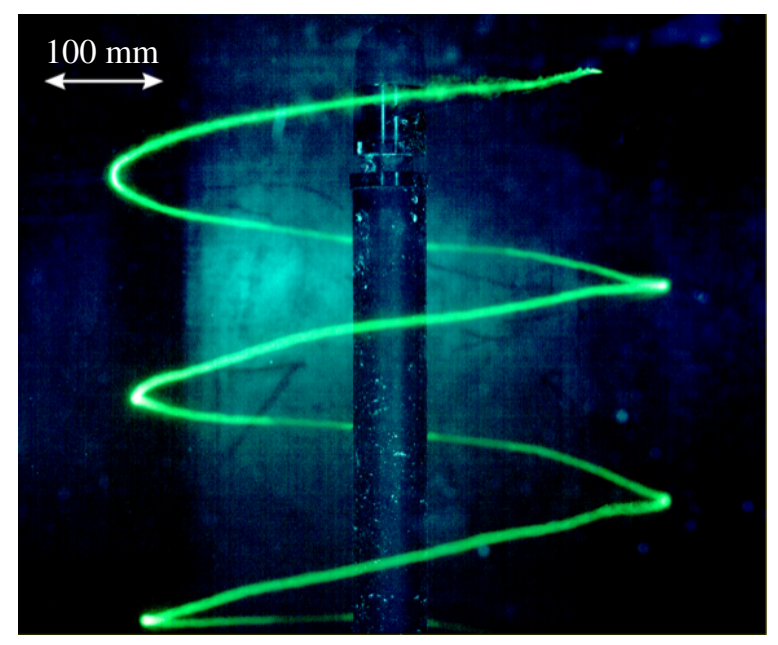

a) Blade with unmodified tip

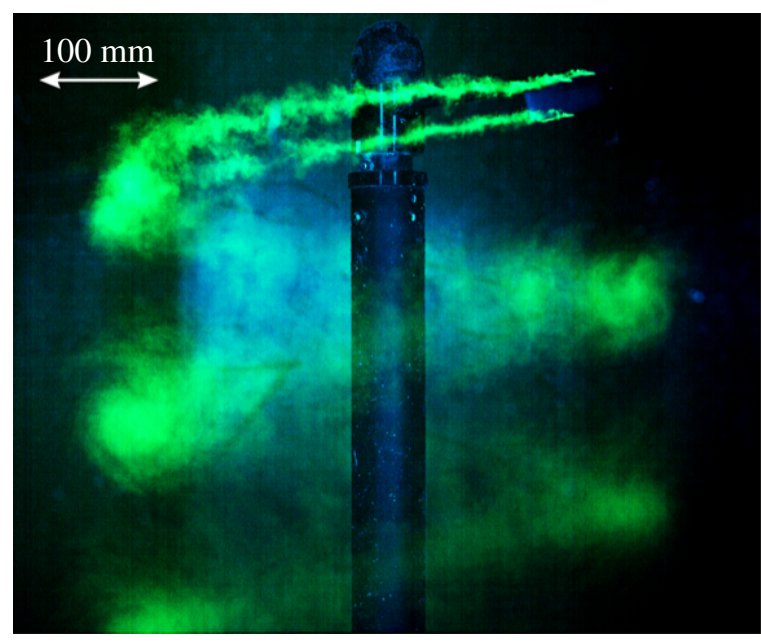

b) Blade with a fin at $d / c=0.2$ and $\alpha_{f}=14^{\circ}$

Fig. 4 Dye visualizations of the tip vortex system.

\section{A. Effect of the tip fin}

A qualitative analysis of the vortex structure and the wake evolution can be obtained from dye visualizations. Figure 4 shows a comparison between tip vortex structures generated by the unmodified blade and by the blade equipped with a fin at a distance $d=0.2 c$ from the tip at an angle of attack $\alpha_{f}=14^{\circ}$. The unmodified blade produces a concentrated vortex, which then forms a helical structure while moving downstream. In unperturbed systems involving a single vortex, the core evolution is expected to be dominated by viscous diffusion inducing only a modest growth. As far as can be judged from the dye pattern in Fig. 4 4 , the core size appears indeed to remain practically unchanged after two revolutions. In the later stages, a disturbance affecting the overall helix geometry is visible. This is caused by slight non-uniformities in the free-stream velocity over the rotor diameter at this extremely low value. Parts of the vortex are transported with slightly higher velocities than the rest, causing the visible deformation of the helix.

The wake is drastically different for the case with fin in Fig. 4p. Two separate vortices of comparable strength are created at the blade and fin tips, and the presence of the additional vortex has a strong effect on the wake development. Both vortices appear to undergo a rapid destabilization immediately after the roll-up, indicated by the perturbed dye filaments in the core regions. After approximately half a turn of the helical pair, the vortices merge into a single structure with what appears to be a significantly wider and more turbulent core. The overall structure of the helix, and in particular its pitch, are nevertheless similar to the case without fin in Fig. 4 4 and appear to be relatively unaffected by the merging process.

The above observations are complemented by stereoscopic PIV measurements. Figures 5 and 6 present phaseaveraged fields of the azimuthal vorticity in the center plane of the rotor near the tip, for the two cases shown in Fig. 4 They were obtained from 250 instantaneous fields measured in FOV B (Fig. 22). Confirming the visual impression acquired from the dye visualizations, the sequence in Fig. 5 shows that the tip vortex retains its concentrated structure with a slowly increasing core size. The vorticity field for the modified blade in Fig. 6 shows that two distinct like-signed vortices of similar are size generated at the tip and the fin, which rotate around each other while moving downstream. A deformation of the vortex structure, combined with a decrease of the core separation, sets in between rotor phases $\gamma=90^{\circ}$ and $180^{\circ}$, resulting in the combination of both vortices into a single one. The field in Fig. 6 $\mathrm{d}$ shows that the size of the merged vortex core is significantly larger than the one for the unmodified blade at the same time, with a strongly reduced vorticity peak.

The measured velocity fields can be analysed further to extract relevant parameters characterizing the tip vortices. The vortex centers can be identified by finding the minima of the $\lambda_{2}$-parameter introduced by Jeong \& Hussain [39]. The separation distance of the two initial vortices for cases with a fin can then be calculated. The swirl velocity profile of a given vortex is obtained by projecting the velocities on a local polar grid $(\rho, \varphi)$ and by averaging the azimuthal component $u_{\varphi}$ for each radius $\rho$ (Fig. 3). A typical example is shown in Fig. 10a. The profile has a maximum at $\rho=a_{\max }$; this distance is a measure for the size of the vortex core. For vortices with a Gaussian vorticity distribution 


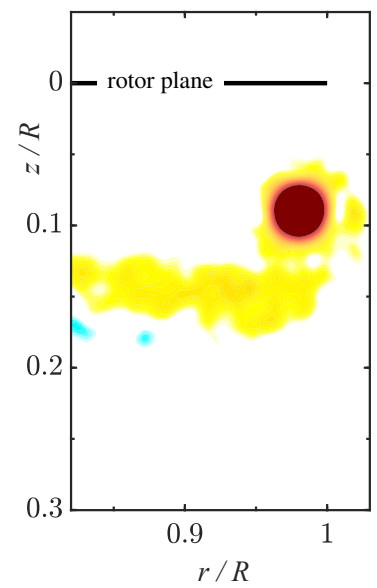

a) $\gamma=36^{\circ}$

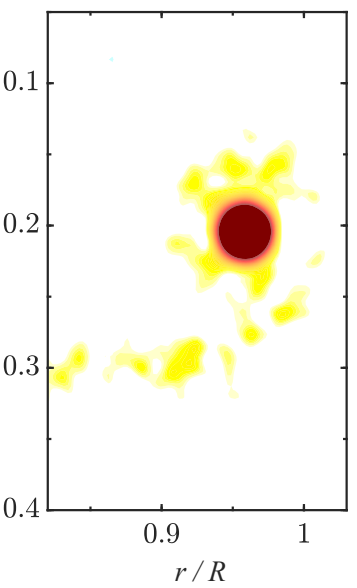

b) $\gamma=90^{\circ}$

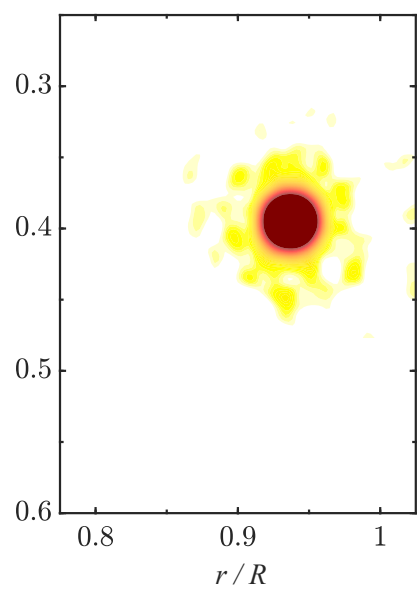

c) $\gamma=180^{\circ}$

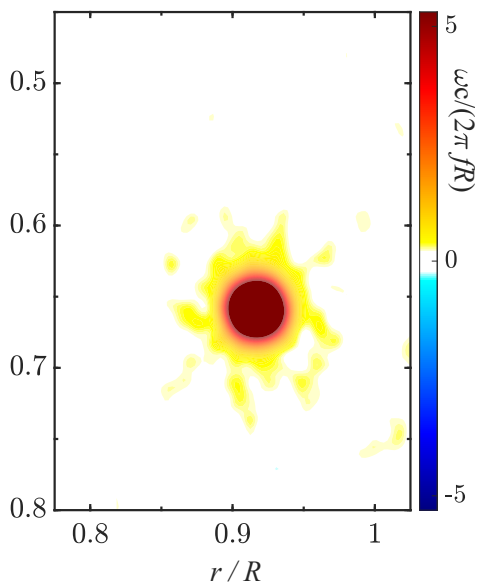

d) $\gamma=306^{\circ}$

Fig. 5 Phase-averaged vorticity in the center plane of the rotor for the unmodified blade.

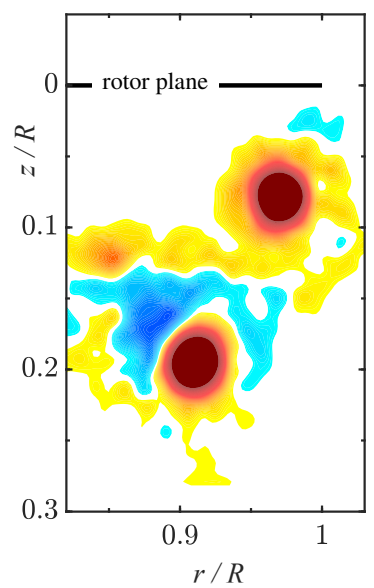

a) $\gamma=36^{\circ}$

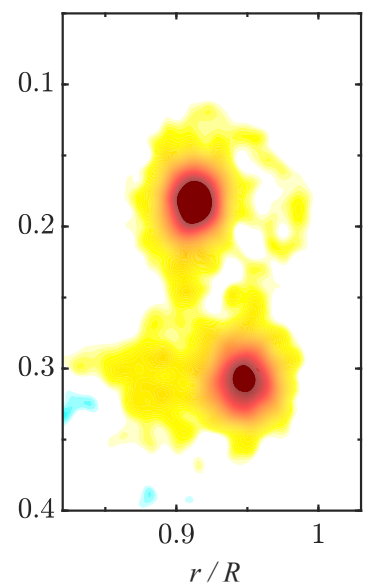

b) $\gamma=90^{\circ}$

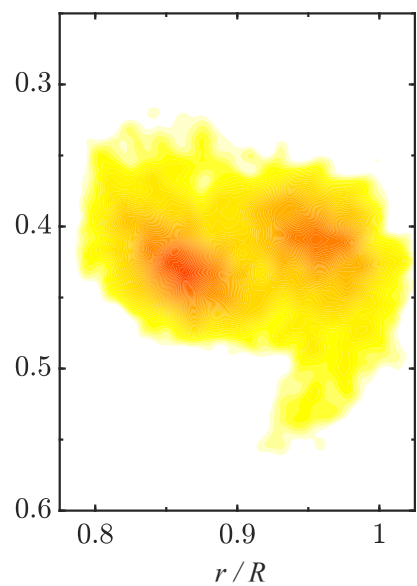

c) $\gamma=180^{\circ}$

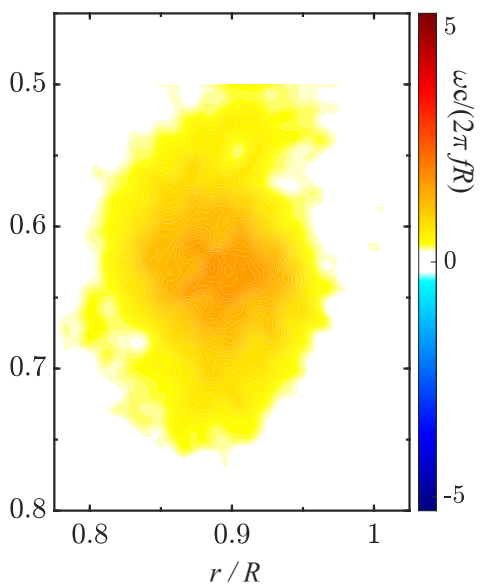

d) $\gamma=306^{\circ}$

Fig. 6 Vorticity fields for the blade with a fin at $d / c=0.2$ and $\alpha_{f}=14^{\circ}$.

(i.e., proportional to $\mathrm{e}^{-\rho^{2} / a^{2}}$ ), $a_{\max }$ is about $12 \%$ larger than the core size parameter $a$. Since many previous theoretical results refer to Gaussian vortices, we here also consider the equivalent Gaussian core size $a=a_{\max } / 1.12$. Finally, the circulation of the vortices is calculated by integrating the velocity on contours surrounding the vortex cores and enclosing all the vorticity that can be attributed to the given vortex.

In Fig. 7, the evolution of the vortex core radii for the cases without and with fin are shown. The dashed lines represent the laminar growth resulting from viscous diffusion of vorticity, as given by Eq. (1). The data for the unperturbed vortex in Fig. $7 \mathrm{~h}$ is in good agreement with the theoretical prediction, indicating a laminar inner core region, despite the high Reynolds number. The core radius increases by a factor of only about 1.3 during one and a half rotor rotations. Since the high velocity gradients in the core persist, a following blade encountering this vortex would cause a strong interaction. For the modified blade, the core evolution in Fig. $7 \mathrm{p}$ reveals a merging process combined with a prior core growth which is much faster than for the single vortex in Fig. 7 k. During the merging process, marked by the grey region, the centers and corresponding core radii cannot be accurately detected for all rotor phases, explaining the missing data points in this interval.

The onset of merging occurs when the vortex core size exceeds a certain fraction of the separation distance. For two-dimensional Gaussian vortices, a critical ratio $a / b=0.24$ was found [6]. Figure 8 a shows the evolution of this parameter for the modified blade. It is similar for both blade tip and fin vortices, and the critical value is reached at a rotor phase of $\gamma \approx 110^{\circ}$. The final vortex resulting from the merging process has a normalised core radius of $a / c \approx 0.25$, 


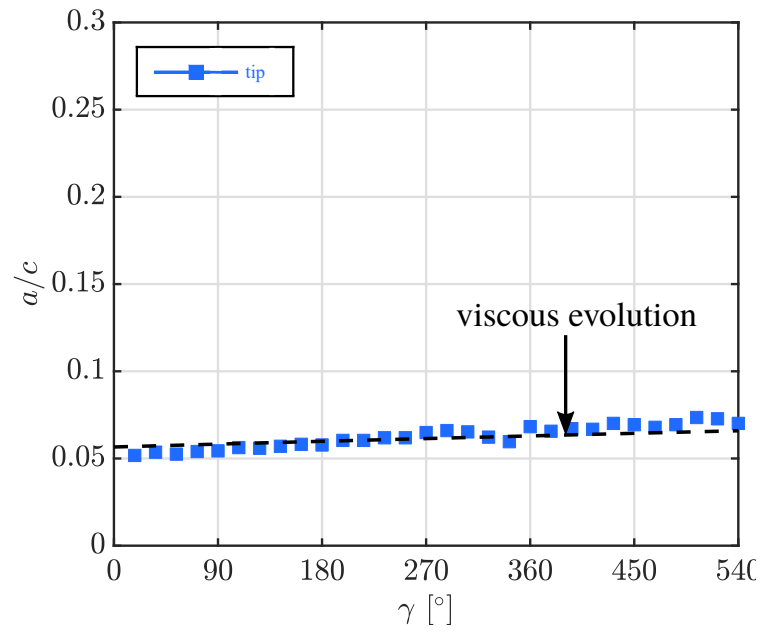

a) Blade without fin

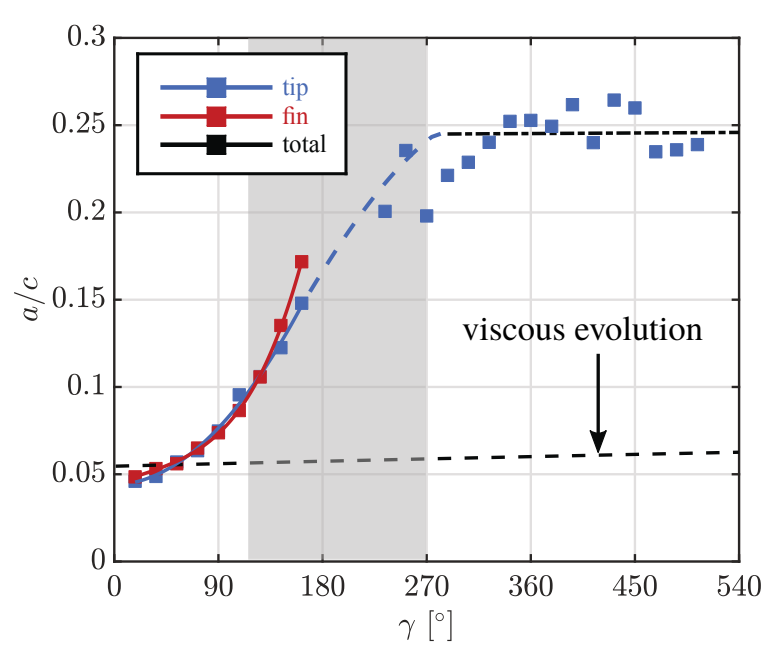

b) Blade with afin at $d / c=0.2$ and $\alpha_{f}=14^{\circ}$

Fig. 7 Comparison of the core size evolution for blades without and with fin. The grey region in b) corresponds to the convective merging phase.

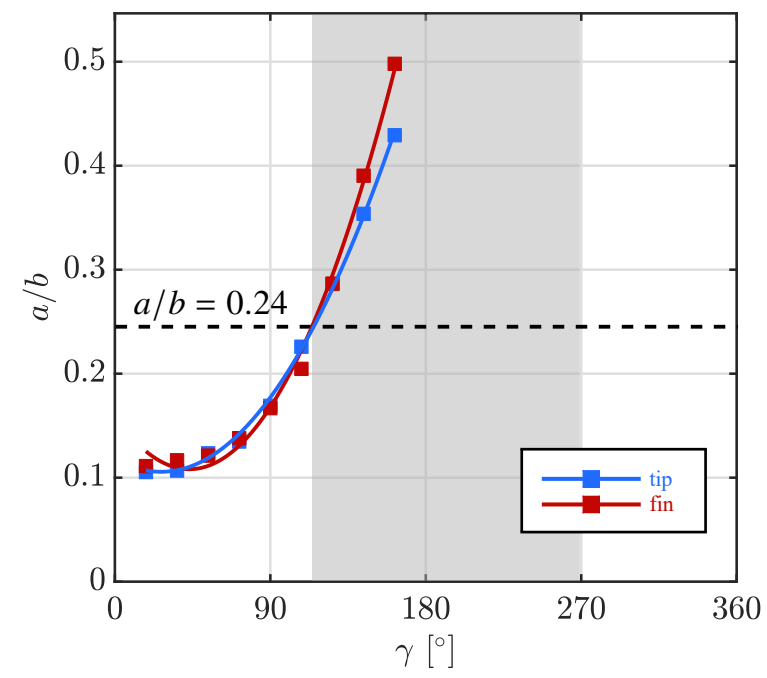

a) Rescaled core size

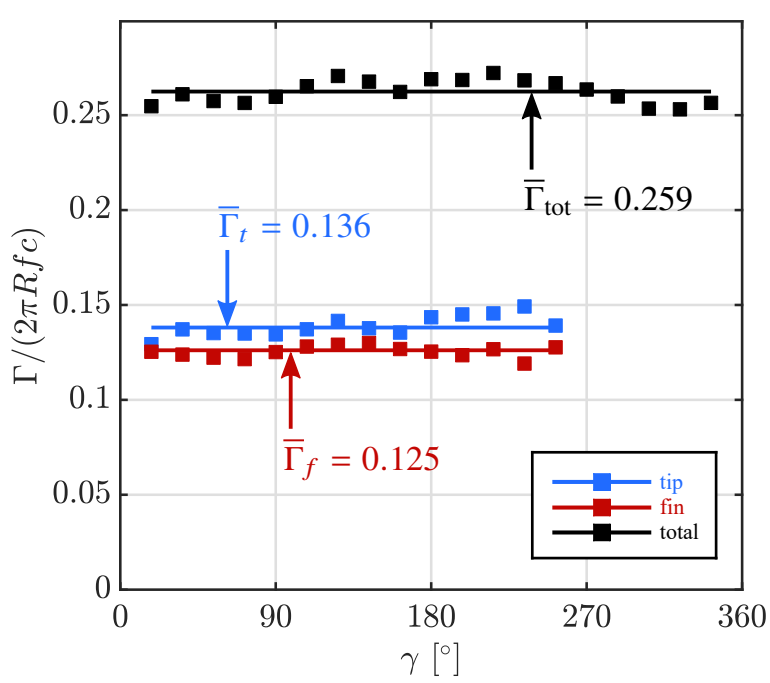

b) Circulation

Fig. 8 Vortex pair parameters relevant for merging, for the rotor blade with a fin at $d / c=0.2$ and $\alpha_{f}=14^{\circ}$. The grey region in a) corresponds to the convective merging phase.

which represents an increase by a factor of 5 from the initial vortex core size, and an almost 4-fold increase with respect to the unmodified blade.

Measurement results of the individual and total circulation of the tip vortices for the blade with a fin are shown in Fig. 8 p. The ratio between the circulations of fin and tip vortices is about 0.9 , which is not far from the optimum value of 1 for complete merging in two dimensions. The fin configuration presented in this section is therefore close to the desired optimum configuration in this study. It should be noted that the total circulation, given by the sum of tip and fin vortex circulations is about $15 \%$ larger than the circulation of the tip vortex without fin. The influence of the presence of the fin on this and other parameters is further examined in Sec. IV.C 


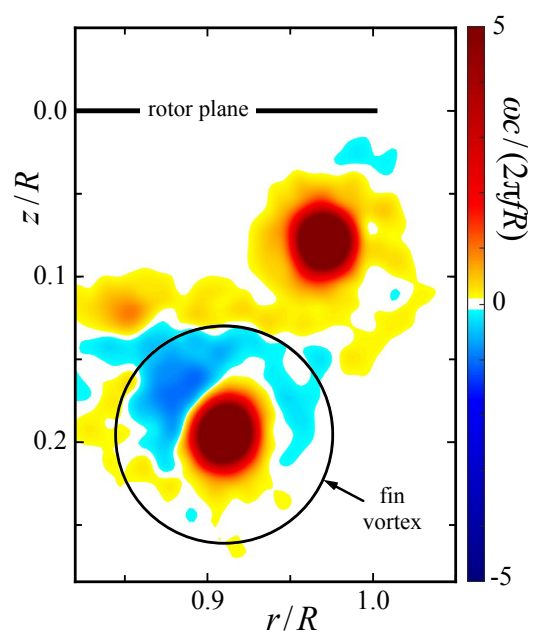

a) Azimuthal vorticity

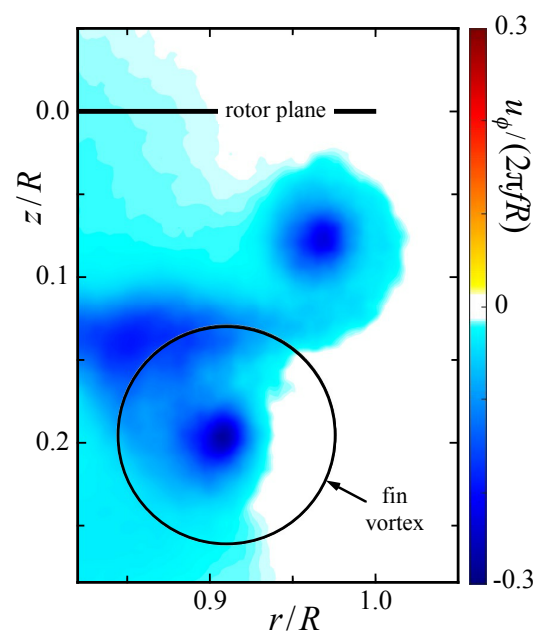

b) Axial velocity

Fig. 9 Flow field in blade tip region, for the case with a fin at $d / c=0.2$ and $\alpha_{f}=14^{\circ}$, at wake age $\gamma=36^{\circ}$.

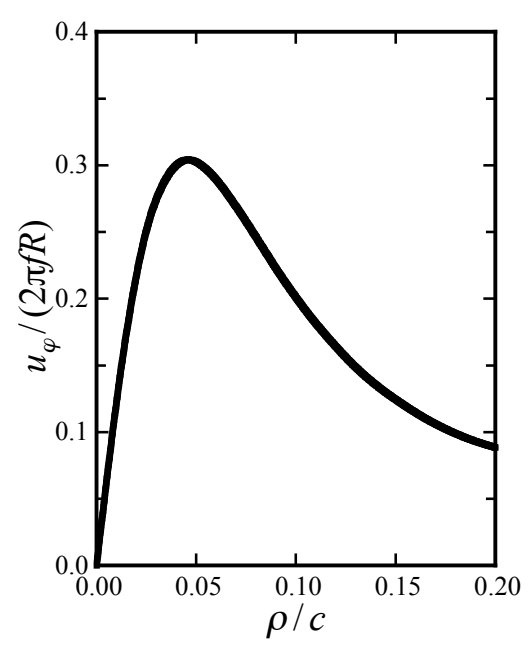

a) Swirl velocity

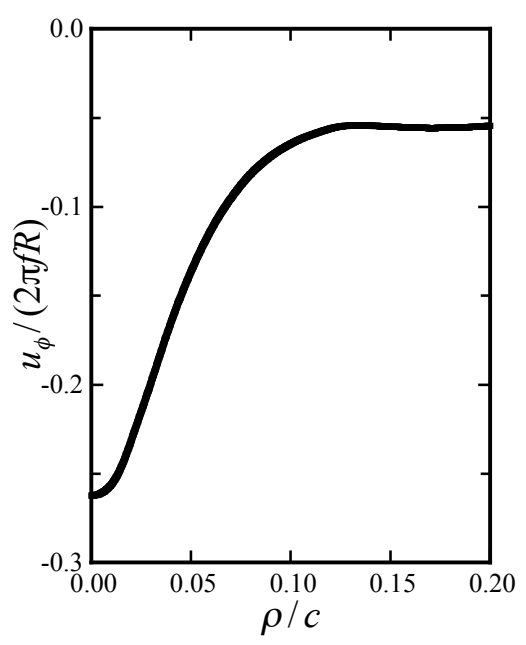

b) Axial core velocity

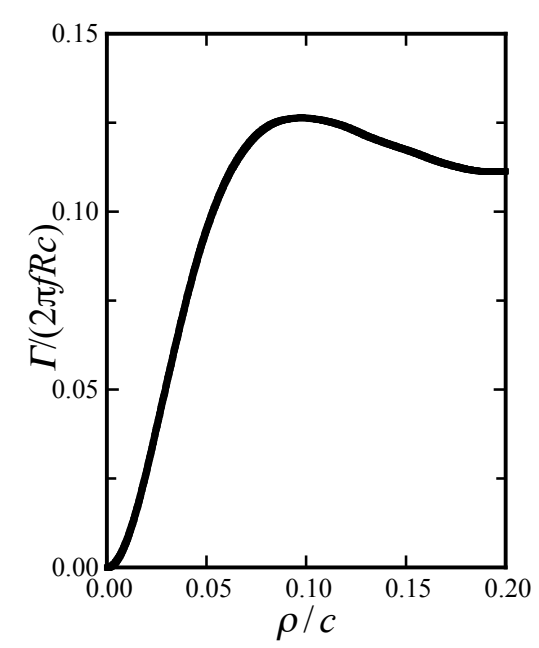

c) Circulation

Fig. 10 Radial profiles of the fin vortex, obtained by averaging the data in Fig. 9 over the local azimuthal angle $\varphi$.

\section{B. Evidence of centrifugal instability}

The experimental measurements have shown that for the case of a finned blade, the cores of both tip vortices increase in size much more rapidly than the one of the single vortex without fin, which roughly follows a viscous diffusion process. This suggests the presence of an instability phenomenon disrupting the vortex cores in this new configuration. Possible candidates are the short-wave elliptic [19, 25] and curvature [26-30] instabilities, which can arise in closely-spaced vortices and significantly affect their core structure. However, estimates of their growth rates for the present configurations, based on previous theoretical work, predict an amplification which is much too weak and incompatible with the observed dynamics.

Another potentially rapid mechanism is centrifugal instability. It arises in vortices whose radial vorticity profile changes sign, which is equivalent to a decrease in absolute circulation with increasing distance from the vortex center. The flow measurements in the near region behind the finned blade indeed show the existence of vorticity of opposite sign in the vicinity of the vortex cores, as shown in Fig. 9 a for the fin configuration discussed in the previous section, at a wake angle of $\gamma=36^{\circ}$. The negative vorticity comes partly from the vorticity layer shed from the inner part of the blade, but it may also be due to the presence of the fin vortex which induces this vorticity on the surface of the blade.

In order to establish a link with centrifugal instability theory, we determine the average profiles of swirl and 


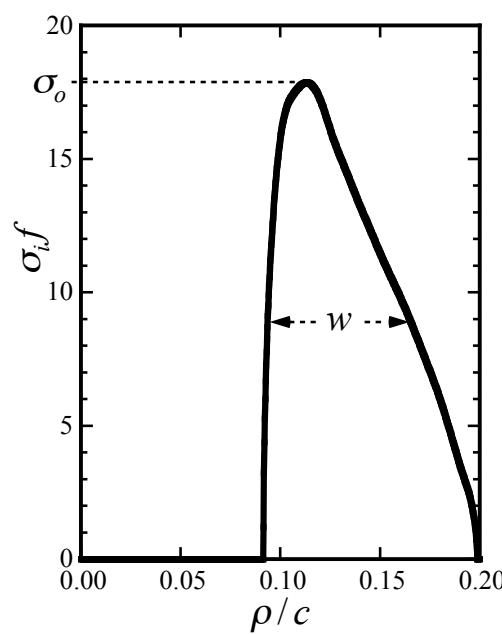

a) Local inviscid growth rate

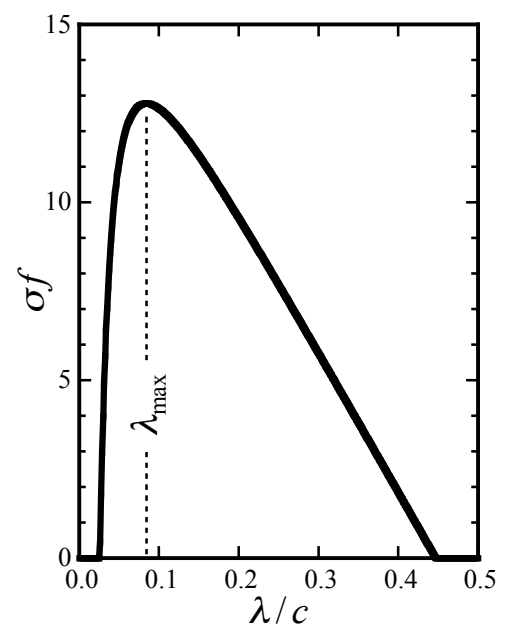

b) Global viscous growth rate

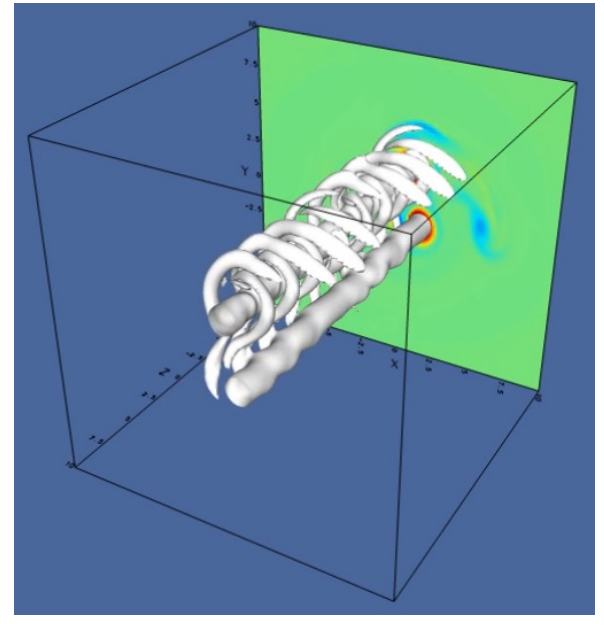

c) Model simulation

Fig. 11 Predictions of centrifugal instability growth rates based on the profiles in Fig. 10 Panel c) shows the result of a numerical simulation of a straight vortex pair with an axially uniform initial condition given by the fields in Fig. 9

axial velocity for the fin vortex in the example shown in Fig. 9. The velocity along the vortex axis is the component perpendicular to the measurement plane, i.e. $u_{\phi}$ in the rotor frame; its distribution is shown Fig. 9p. The profiles were obtained as described before, by transforming the velocity components in the $z-r$ plane to a local polar coordinate system $\rho-\varphi$ around the vortex center, and then averaging on circles of radius $\rho$. The results are plotted in Fig. 10, showing the familiar swirl velocity profile, as well as an axial velocity deficit in the vortex core. The circulation is given by $\Gamma=2 \pi \rho u_{\varphi}$. Its profile in Fig. 10 p shows a decrease for $0.1<\rho / c<0.2$, i.e. in the region between twice and four times the core radius, which is a sufficient condition for centrifugal instability.

We can estimate the instability growth rate by considering theoretical results for axisymmetric vortices having the profiles in Fig. 10. According to Billant \& Gallaire [34], the local inviscid growth rate for centrifugal instability is given by

$$
\sigma_{i}(\rho)=\mathfrak{R}\left[-2 \rho \Omega \Omega^{\prime} \frac{\rho \Omega^{\prime} \omega+u_{\phi}^{\prime 2}}{\rho^{2} \Omega^{\prime 2}+u_{\phi}^{\prime 2}}\right]^{1 / 2},
$$

where $\Omega=u_{\varphi} / \rho, \omega=u_{\varphi}^{\prime}+u_{\varphi} / \rho$ and the prime (') denotes the radial derivative ( $\left.\mathrm{d} / \mathrm{d} r\right)$. The result is shown in Fig. 11, the growth rate is indeed positive in the region of decreasing circulation. Following Bayly [33], one can use the maximum $\sigma_{o}$ of the local inviscid growth rate and the width $w$ of the radial profile at half-height to estimate a global inviscid growth rate for perturbations of axial wavelength $\lambda$ as $\sigma_{o}[1-\lambda /(2 \pi w)]$. In addition, for viscous flow, a damping term can be estimated as $-v(2 \pi / \lambda)^{2}$, so that the final estimation for the growth rate is

$$
\sigma(\lambda)=\sigma_{o}[1-\lambda /(2 \pi w)]-v(2 \pi / \lambda)^{2} .
$$

The result in Fig. $11 \mathrm{p}$ predicts a most amplified wavelength $\lambda_{\max }$ of roughly $1 \mathrm{~cm}$, which is of the same order as the perturbation scales seen in the visualisations. The corresponding growth rate, normalised with the rotation period of the rotor, is $\sigma f \approx 13$, which represents a growth factor of $O\left(10^{5}\right)$ per revolution. This high value is compatible with the observed rapid growth of of the vortex perturbations and core size.

A further illustration of the centrifugal instability mechanism in our configuration was obtained by a three-dimensional direct numerical simulation of a straight vortex pair flow, whose initial velocity field is uniform along the vortex axes and given by the distributions in Fig. 9 The snapshot in Fig. 11, shows vortical structures at a time corresponding to a wake age $\gamma \approx 150^{\circ}$. Secondary vortices appear in the outer layers of the fin vortex, with a wavelength similar to the theoretical prediction and the experimental observation. This behaviour is in agreement with what is expected for a centrifugal instability. 


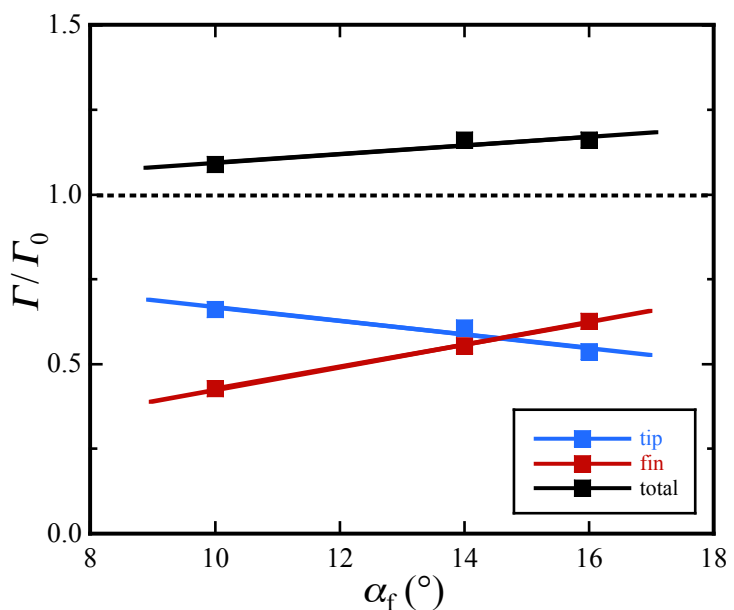

a) Circulation $\left(\Gamma_{0}:\right.$ circulation without fin)

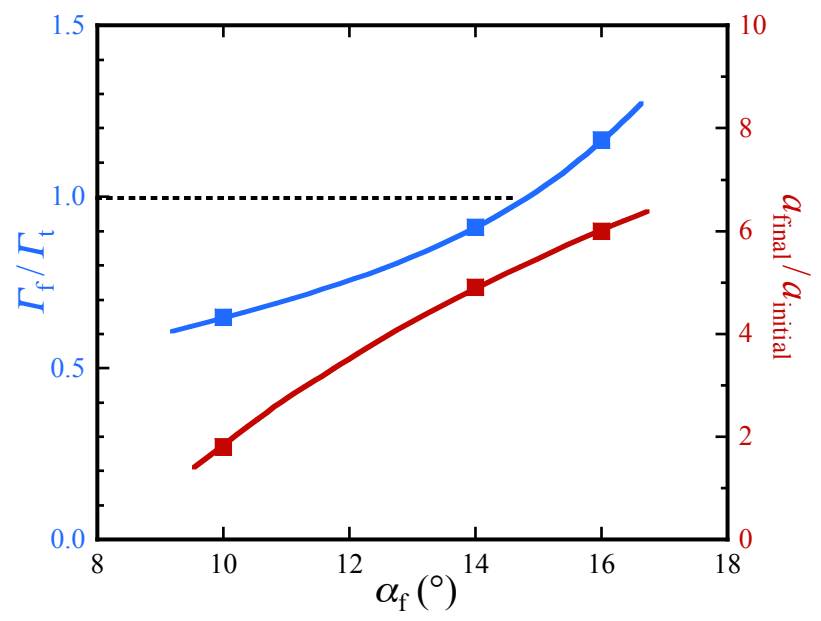

b) Circulation ratio and merged core radius

Fig. 12 Influence of the fin angle $\alpha_{f}$ on vortex circulations and final core size, for $d / c=0.2$.

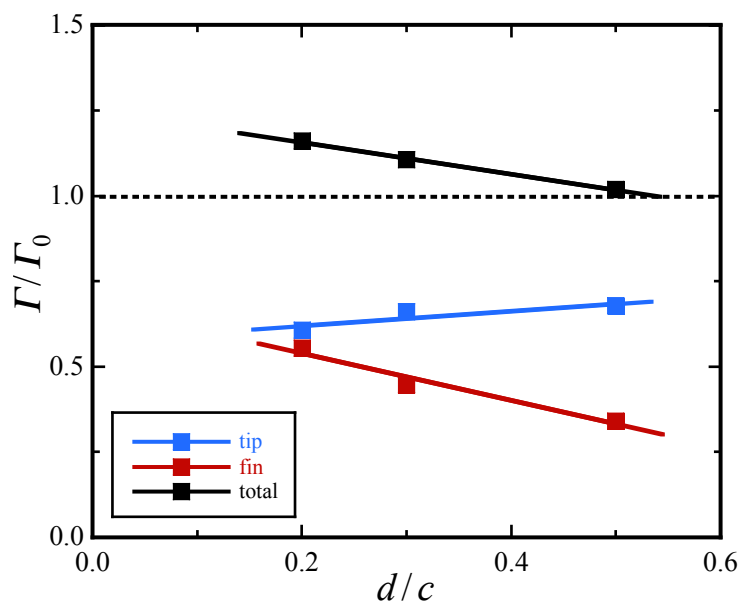

a) Circulation $\left(\Gamma_{0}\right.$ : circulation without fin)

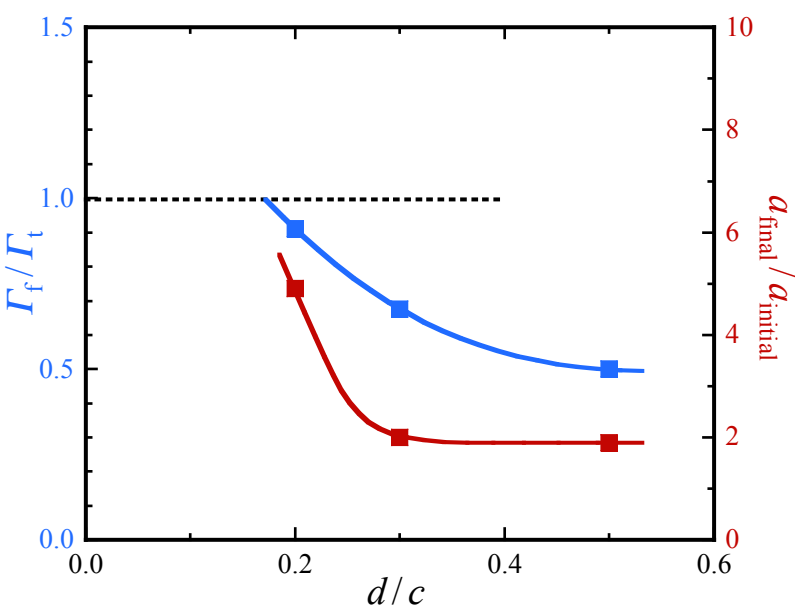

b) Circulation ratio and merged core radius

Fig. 13 Influence of the fin position $d$ on vortex circulations and final core size, for $\alpha_{f}=14^{\circ}$.

\section{Influence of fin parameters}

Varying the parameters defining the fin geometry and position (see Fig. 11) has an effect on the properties of the initial vortex pair, in particular the circulation ratio and the vortex spacing. We here present the results for the variation of two parameters, the fin angle of attack $\alpha_{f}$ and the distance $d$ of the fin to the blade tip, all other parameters being held constant.

The fin angle $a_{f}$ was varied between $10^{\circ}$ and $16^{\circ}$ for a fin position $d / c=0.2$. Increasing this angle is expected to generate stronger fin vortices, which can be used to adjust the circulation ratio to values close to 1 . The measurements in Fig. 12 confirm this tendency. The fin vortex circulation grows linearly with increasing fin angles, and one observes a simultaneous decrease of the primary tip vortex circulation, indicating that an increasing fraction of the circulation generated by the blade is accumulated in the fin vortex. For all angles, the total circulation is slightly higher than for the unmodified case (Fig. 12 ). The corresponding ratios of fin vortex circulation $\Gamma_{f}$ and tip vortex circulation $\Gamma_{t}$, as well as the core growth factors resulting from the merging, are shown in Fig. $12 \mathrm{p}$. For $\alpha_{f}=10^{\circ}$, we find $\Gamma_{f} / \Gamma_{t} \approx 0.6$, leading to a growth factor close to 2 . The circulation ratio increases with $\alpha_{f}$, reaching values close to, and even above, 1 for $\alpha_{f} \geq 14^{\circ}$. For these cases, a very large increase of the core radius (by a factor 5-6) is found after merging.

Figure 13 shows the same vortex parameters as function of the fin position $d$, for a constant fin angle $\alpha_{f}=14^{\circ}$. Moving the fin away from the blade tip leads to a decrease of the fin vortex circulation, despite the constant fin angle, showing that the local flow conditions in the tip region (varying flow direction) have a marked impact on the initial 


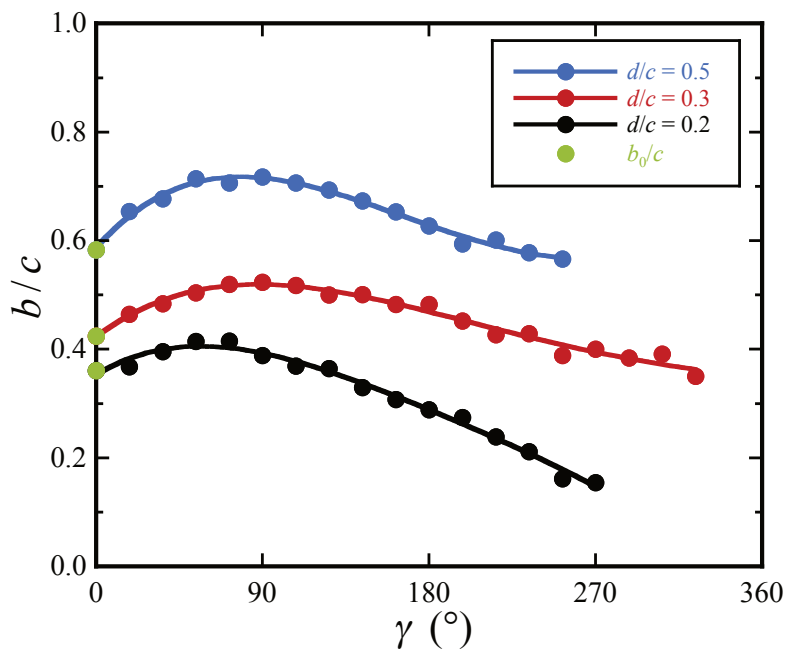

Fig. 14 Evolution of the vortex spacing for different fin positions. $b_{0}$ is the distance between the blade and fin tips.

circulation values. The circulation of the blade tip vortex is also influenced by the fin position: as before, its evolution is opposite to the one of the fin vortex. The total circulation is again higher than for the blade without fin, but it decreases towards this value as the distance between fin and blade tip increases. The resulting circulation ratio $\Gamma_{f} / \Gamma_{t}$ decreases from 0.9 at $d / c=0.2$ to about 0.5 for a fin distance $d / c=0.5$ (Fig. 13p). The lower values of the circulation ratio lead again to a smaller increase of the core radius for the merged vortex.

The fin position with respect to the blade tip has a direct influence on the separation distance of the initial vortex pair, as illustrated in Fig. 14 . In all cases, the initial separation is close to the geometrical distance $b_{0}=\sqrt{d^{2}+h^{2}}$ between blade tip and fin tip. It increases by about $20 \%$ up to a rotor phase $\gamma=90^{\circ}$, before decreasing again up to the merging. Whereas for the higher tip distances $d$ (lower circulation ratios), the vortex separations remain close to $b_{0}$ before merging, a significant decrease to about $50 \%$ of $b_{0}$ is observed for the case with lower $d$ and a circulation ratio closer to 1 .

\section{Two merging scenarios}

From the results of the parameter exploration in the previous section, it appears that the evolution of the co-rotating vortex pair generated by the finned blade tip occurs in basically two qualitatively different ways, each leading to distinct properties of the merged vortex. In particular, the core radius of the final vortex is lower for initial vortex systems in which the fin vortex is considerably weaker than the tip vortex. In these cases, the merging process between the two vortex cores is only partial. Dritschel \& Waugh [40] and Brand \& Nomura [41] have described various types of two-dimensional merging of non-identical vortices. They also find that partial merging of vortices with different circulations generates a smaller final core radius compared to vortex systems with similar strength.

Figure 15 gives a visual impression of the two types of merging. In the case of a complete merging in Fig. $15 \mathrm{a}$, both initial cores are strongly perturbed and mix into a single large-scale turbulent structure. In Fig. 15p, the blade tip vortex keeps its concentrated core structure throughout the merging process, and only the fin vortex disintegrates and is wrapped around the tip vortex. This results in a merged vortex with a much smaller core than in Fig. 15 a.

The two merging scenarios can further be illustrated by the evolution of the vorticity. Another case of complete merging was presented in Sec. IV.A and the corresponding vorticity fields are shown in Fig. 6, Figure 16 contains a sequence depicting a partial merging of the tip vortex pair. It shows that the tip and fin vortices evolve differently. The fin vortex destabilises rapidly, apparently again under the influence of a centrifugal instability - the nearby opposite-signed vorticity is clearly visible in Fig. 16. The tip vortex remains practically unchanged over half of the first rotor rotation, and still appears as a separate structure after one rotation (Figure $16 \mathrm{~d}$ ).

The distinction between two different wake behaviors is also found in other measurements. When the vortex pair separation distance $b$ in Fig. 14 is normalised by the initial separation $b_{0}$, two sets of curves appear, as shown in Fig. 17 A. As noted before, complete merging is accompanied by a faster and stronger decrease of the vortex separation. The previous results have highlighted the importance of the circulations ratio of the initial vortex pair for the further 


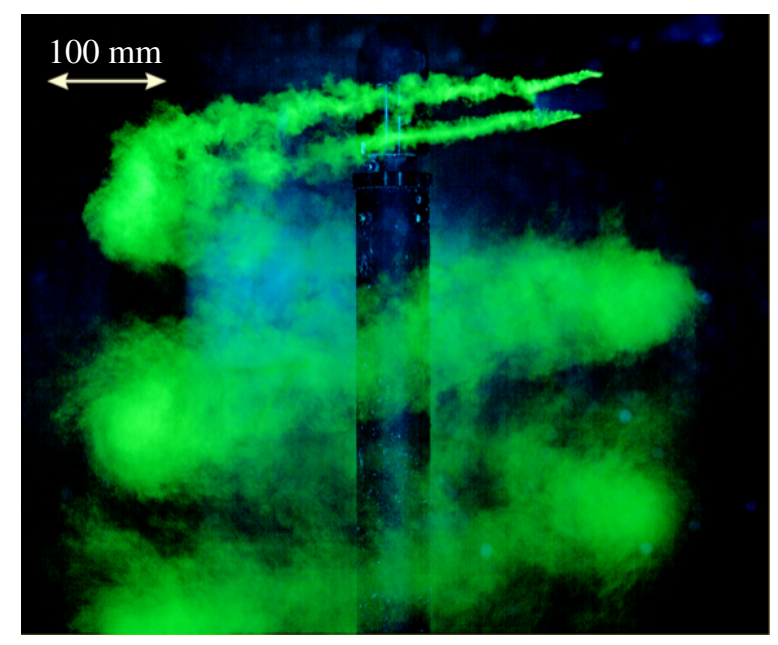

a) Complete merging for $d / c=0.2$ and $\alpha_{f}=16^{\circ}$

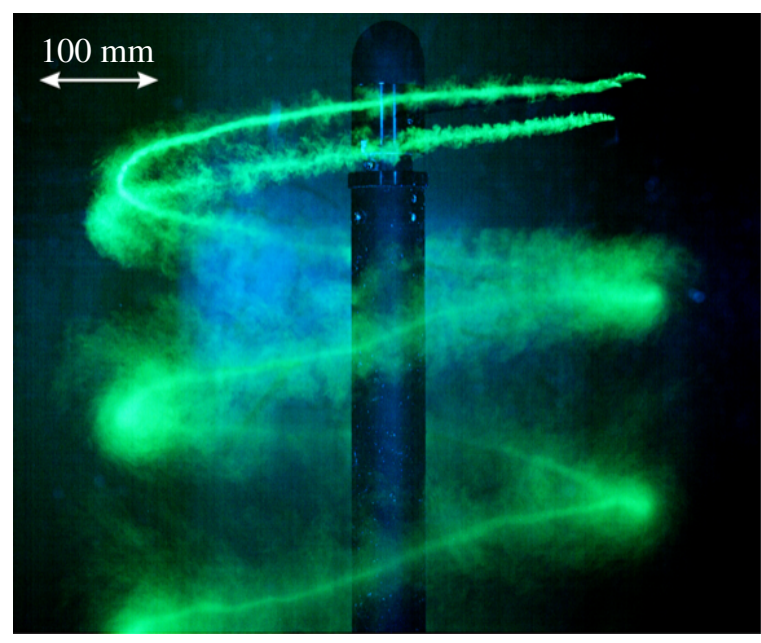

b) Partial merging for $d / c=0.3, \alpha_{f}=10^{\circ}$

Fig. 15 Dye visualizations comparing the two merging scenarios.

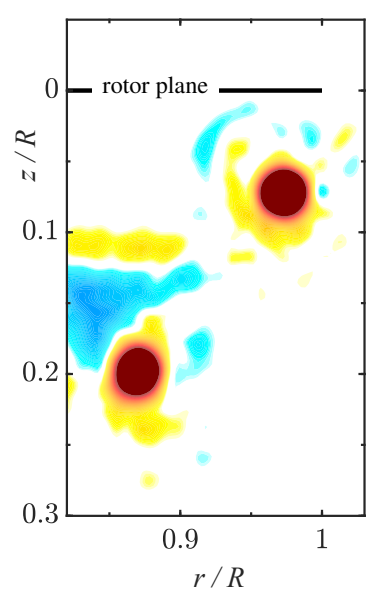

a) $\gamma=36^{\circ}$

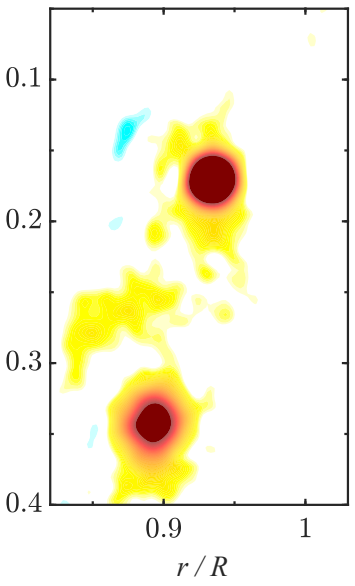

b) $\gamma=90^{\circ}$

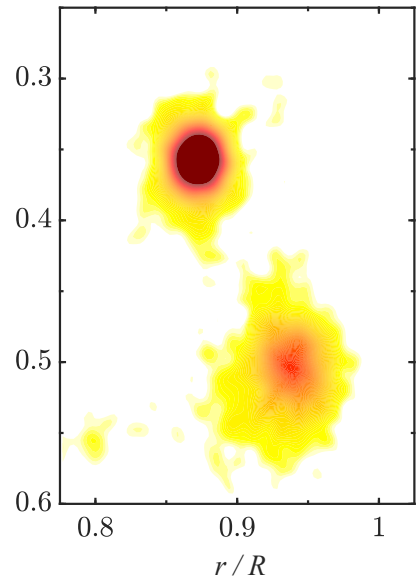

c) $\gamma=180^{\circ}$

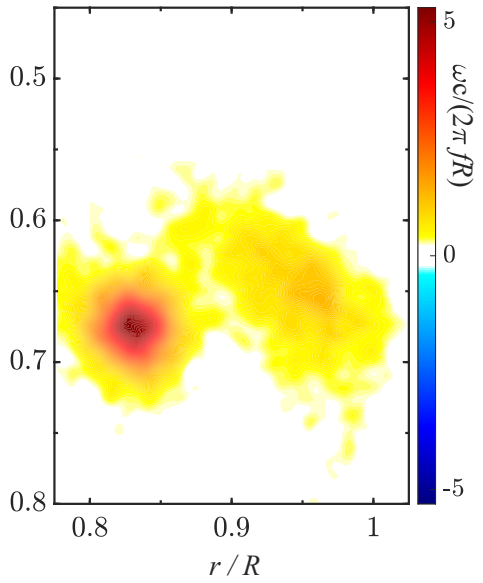

d) $\gamma=306^{\circ}$

Fig. 16 Vorticity fields for the blade with a fin at $d / c=0.3$ and $\alpha_{f}=14^{\circ}$.

evolution of the wake and the size of the final vortex core. In Fig. $17 \mathrm{p}$, the final core radius is plotted as function of the circulation ratio for all cases discussed above. It is here normalised by the initial vortex spacing, for better comparison. The two regimes of vortex interaction appear clearly in this graph. For $\Gamma_{f} / \Gamma_{t} \lesssim 0.8$, partial merging leads only to a small increase of the core radius, whereas for ration above this value, the core growth is 3 to 4 times larger.

\section{E. Counter-rotating vortices}

The merging phenomenon of two vortices into a single one is in principle only observed for co-rotating vortex systems. However, the dynamics and interactions of counter-rotating vortices may also be worth investigating in the context of rotor wakes, as illustrated in Fig. 18. Dye visualizations for two cases where the blade is equipped with a fin at a negative angle $\alpha_{f}$ are shown, generating a counter-rotating initial vortex pair. The fin vortex appears to be weaker, indicated by the rapidly disturbed dye in its core regions for the early wake stages. It is deformed and ultimately wrapped up around the stronger tip vortex. After one revolution, the concentrated structure of the tip vortex cannot be observed anymore, indicating that the interaction between the two vortices may have an influence on the core evolution. These qualitative impressions require a more quantitative analysis through PIV measurements, which is currently underway. 


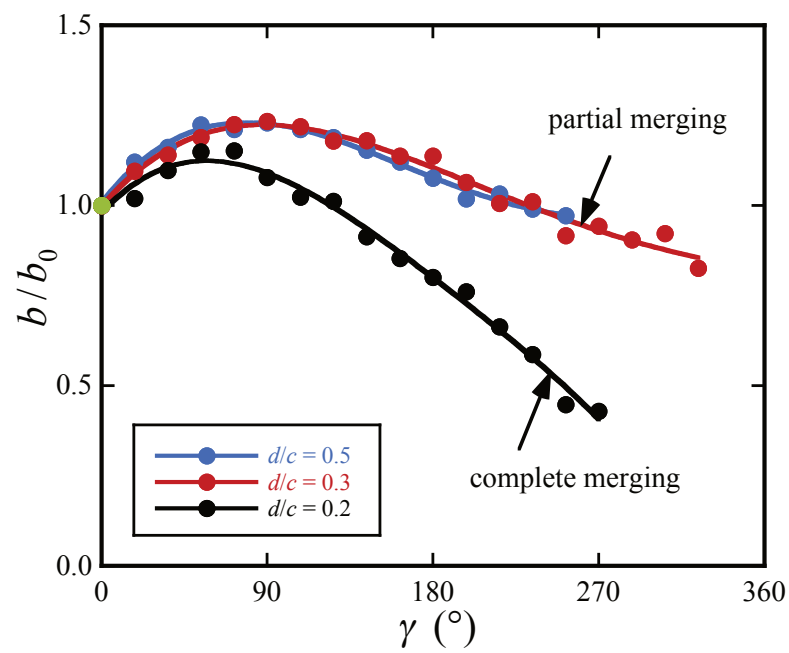

a) Evolution of the separation distance

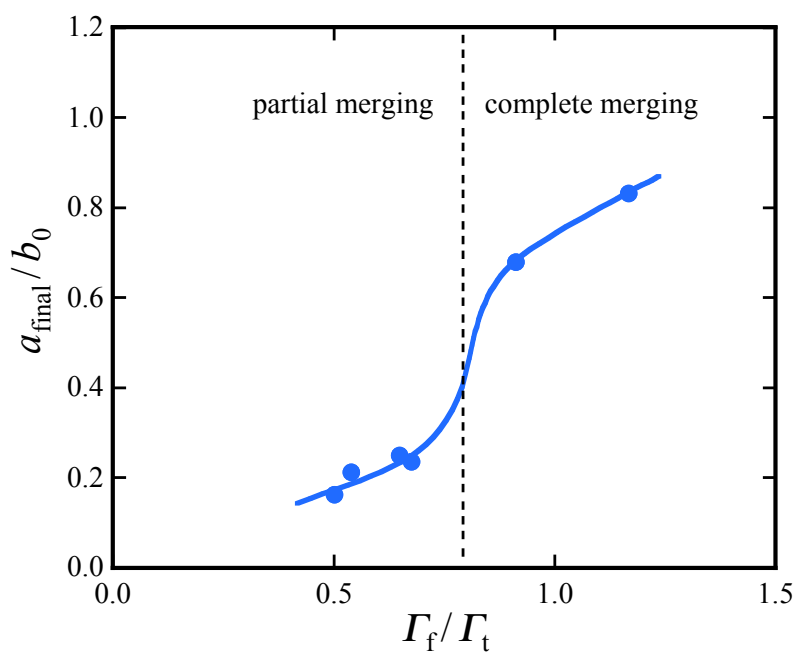

b) Final vortex size as function of circulation ratio

Fig. 17 Vortex parameters characterizing the two merging scenarios.

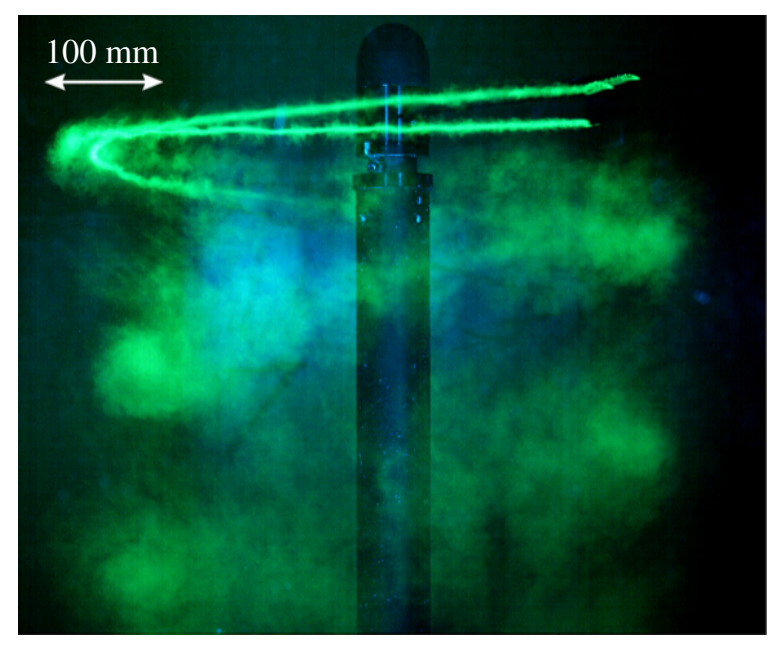

a) Blade with a fin at $d / c=0.3$ and $\alpha_{f}=-14^{\circ}$

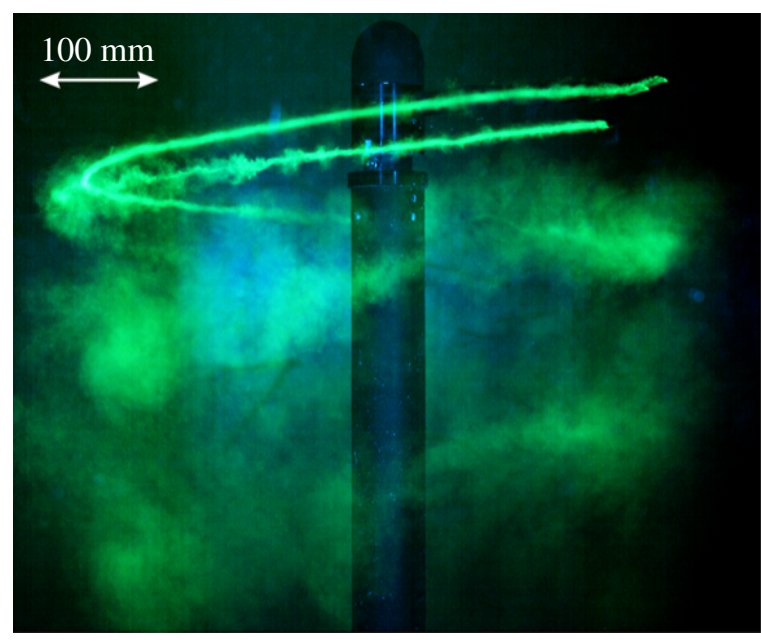

b) Blade with a fin at $d / c=0.5$ and $\alpha_{f}=-14^{\circ}$

Fig. 18 Visualizations of counter-rotating tip vortex pairs obtained with negative fin angles.

\section{Conclusion}

We have presented experimental observations and measurements concerning the dynamics of helical pairs of co-rotating vortices in the wake of a single-bladed rotor. They were generated by equipping the rotor blade with a perpendicular fin placed on the pressure side near the tip. By choosing appropriate values for the fin's geometrical and position parameters, it is possible to generate pairs of vortices of nearly equal strength. Dye visualizations and velocity measurements in a water channel have shown that these vortices merge into a single final vortex within one blade revolution. This rapid merging occurs because of a surprisingly strong growth of the vortex cores of the initial pair. The analysis of the velocity field revealed evidence for a three-dimensional centrifugal-type instability responsible for this behavior.

Variations of the fin parameters (position, angle of attack) lead to different properties of the initial vortex pair, such as separation distance and circulation ratios. The different wake evolutions can be divided into two main scenarios, depending primarily on the circulation ratio of the pair. In addition to the fast and complete merging for ratios of fin and tip vortex circulations greater than approximately 0.8 , partial merging was observed for lower values. Whereas partial merging resulted in only a moderate increase of the vortex core radius after merging, a core growth by factors exceeding 
5 were found for complete merging. A remarkable observation is the fact that the initial vortex separation alone (i.e., the distance between blade tip and fin) is not a determining parameter for the final core size. Even for small distances, a very large core growth can be obtained, provided that the circulation ratio is close to 1 .

The experiments in this study have shown that it is possible to achieve a significant expansion of the tip vortex core of a rotor blade by adding an appropriately sized fin near the blade tip. The generation of a co-rotating helical vortex pair, followed by a rapid destabilisation through a centrifugal instability and a complete merging of the vortex cores, produces a final enlarged vortex within less than one rotation of the blade, i.e. within about 15 chord lengths behind the trailing edge in our configuration. These results may therefore be of interest in the context of blade-vortex interaction mitigation, where a rapid reduction of the strong velocity gradients associated with concentrated tip vortex cores is needed.

\section{Acknowledgments}

This work is part of the German-French project TWIN-HELIX, supported by the Deutsche Forschungsgemeinschaft (grant no. 391677260) and the French Agence Nationale de la Recherche (grant no. ANR-17-CE06-0018). The authors are grateful to Andrés Castillo Castellanos for the simulation result in Fig.11.c).

\section{References}

[1] Yung, Y., "Rotor blade-vortex interaction noise," Progress in Aerospace Sciences, Vol. 36, No. 2, 2000 , pp. 97-115. https://doi.org/10.1016/S0376-0421(99)00012-3

[2] Zhao, R., Shen, W., Knudsen, T., and Bak, T., "Fatigue distribution optimization for offshore wind farms using intelligent agent control," Wind Energy, Vol. 15, No. 7, 2012, pp. 927-944. https://doi.org/10.1002/we.1518

[3] Schmitz, F. H., and Yung, Y. H., "Helicopter impulsive noise: theoretical and experimental status," Journal of Sound and Vibration, Vol. 109, No. 3, 1986, pp. 361-422. https://doi.org/10.1016/S0022-460X(86)80378-9

[4] Yung, Y., Gmelin, B., Splettstoesser, W., Philippe, J. J., Prieur, J., and Brooks, T. F., "Reduction of helicopter blade-vortex interaction noise by active rotor control technology," Progress in Aerospace Sciences, Vol. 33, No. 9-10, 1997, pp. 647-687. https://doi.org/10.1016/S0376-0421(97)00006-7

[5] Brocklehurst, A., and Barakos, G. N., "A review of helicopter rotor blade tip shapes," Progress in Aerospace Sciences, Vol. 56, 2013, pp. 35-74. https://doi.org/10.1016/j.paerosci.2012.06.003

[6] Meunier, P., Le Dizès, S., and Leweke, T., "Physics of vortex merging," Comptes Rendus Physique, Vol. 6, No. 4-5, 2005 , pp. 431-450. https://doi.org/10.1016/j.crhy.2005.06.003

[7] Leweke, T., Le Dizès, S., and Williamson, C. H., "Dynamics and instabilities of vortex pairs," Annual Review of Fluid Mechanics, Vol. 48, 2016, pp. 507-541. https://doi.org/10.1146/annurev-fluid-122414-034558.

[8] Cerretelli, C., and Williamson, C. H. K., “The physical mechanism for vortex merging," Journal of Fluid Mechanics, Vol. 475, 2003, pp. 41-77. https://doi.org/10.1017/S0022112002002847

[9] Melander, M. V., Zabusky, N. J., and McWilliams, J. C., "Symmetric vortex merger in two dimensions: causes and conditions," Journal of Fluid Mechanics, Vol. 195, 1988, pp. 303-340. https://doi.org/10.1017/S0022112088002435

[10] Leweke, T., Quaranta, H. U., Bolnot, H., Blanco-Rodríguez, F. J., and Le Dizès, S., "Long- and short-wave instabilities in helical vortices," Journal of Physics: Conference Series, Vol. 524, 2014, Paper 012154.

[11] Widnall, S. E., “The stability of a helical vortex filament,” Journal of Fluid Mechanics, Vol. 54, No. 4, 1972 , pp. $641-663$. https://doi.org/10.1017/S0022112072000928

[12] Gupta, B. P., and Loewy, R. G., "Theoretical analysis of the aerodynamic stability of multiple, interdigitated helical vortices," AIAA Journal, Vol. 12, No. 10, 1974, pp. 1381-1387. https://doi.org/10.2514/3.49493

[13] Kawada, S., "Induced velocity by helical vortices," Journal of the Aeronautical Sciences, Vol. 1936, No. 3, 1936, pp. 86-87. https://doi.org/10.2514/8.141

[14] Hardin, J. C., "The velocity field induced by a helical vortex filament," The Physics of Fluids, Vol. 25, No. 11, 1982, pp. 1949-1952. https://doi.org/10.1063/1.863684 
[15] Okulov, V. L., "On the stability of multiple helical vortices," Journal of Fluid Mechanics, Vol. 521, 2004 , pp. 319-342. https://doi.org/10.1017/S0022112004001934

[16] Quaranta, H. U., Bolnot, H., and Leweke, T., "Long-wave instability of a helical vortex," Journal of Fluid Mechanics, Vol. 780, 2015, pp. 687-716. https://doi.org/10.1017/jfm.2015.479

[17] Quaranta, H. U., Brynjell-Rahkola, M., Leweke, T., and Henningson, D. S., "Local and global pairing instabilities of two interlaced helical vortices," Journal of Fluid Mechanics, Vol. 863, 2019, pp. 927-955. https://doi.org/10.1017/jfm.2018.904

[18] Moore, D. W., and Saffman, P. G., “The instability of a straight vortex filament in a strain field," Proceedings of the Royal Society A, Vol. 346, No. 1646, 1975, pp. 413-425. https://doi.org/10.1098/rspa.1975.0183

[19] Kerswell, R. R., "Elliptical instability," Annual Review of Fluid Mechanics, Vol. 34, 2002, pp. 83-113. https://doi.org/10.1146/ annurev.fluid.34.081701.171829

[20] Leweke, T., and Williamson, C. H. K., “Cooperative elliptic instability of a vortex pair," Journal of Fluid Mechanics, Vol. 360, 1998, pp. 85-119. https://doi.org/10.1017/S0022112097008331

[21] Meunier, P., and Leweke, T., "Elliptic instability of a co-rotating vortex pair," Journal of Fluid Mechanics, Vol. 533, 2005, pp. 125-159. https://doi.org/10.1017/S0022112005004325

[22] Roy, C., Schaeffer, N., Le Dizès, S., and Thompson, M., "Stability of a pair of co-rotating vortices with axial flow," Physics of Fluids, Vol. 20, No. 9, 2008, Paper 094101. https://doi.org/10.1063/1.2967935

[23] Roy, C., Leweke, T., Thompson, M. C., and Hourigan, K., "Experiments on the elliptic instability in vortex pairs with axial core flow," Journal of Fluid Mechanics, Vol. 677, 2011, pp. 383-416. https://doi.org/10.1017/jfm.2011.91

[24] Blanco-Rodríguez, F. J., Le Dizès, S., Selçuk, C., Delbende, I., and Rossi, M., "Internal structure of vortex rings and helical vortices," Journal of Fluid Mechanics, Vol. 785, 2015, pp. 219-247. https://doi.org/10.1017/jfm.2015.631

[25] Blanco-Rodríguez, F. J., and Le Dizès, S., "Elliptic instability of a curved Batchelor vortex," Journal of Fluid Mechanics, Vol. 804, 2016, pp. 224-247. https://doi.org/10.1017/jfm.2016.533.

[26] Fukumoto, Y., and Hattori, Y., "Curvature instability of a vortex ring," Journal of Fluid Mechanics, Vol. 526, 2005 , pp. 77-115. https://doi.org/10.1017/S0022112004002678.

[27] Hattori, Y., and Fukumoto, Y., "Short-wave stability of a helical vortex tube: the effect of torsion on the curvature instability," Theoretical and Computational Fluid Dynamics, Vol. 24, 2010, pp. 363-368. https://doi.org/10.1007/s00162-009-0145-2

[28] Hattori, Y., and Fukumoto, Y., "Modal stability analysis of a helical vortex tube with axial flow," Journal of Fluid Mechanics, Vol. 738, 2014, pp. 222-249. https://doi.org/10.1017/jfm.2013.591

[29] Blanco-Rodríguez, F. J., and Le Dizès, S., "Curvature instability of a curved Batchelor vortex,” Journal of Fluid Mechanics, Vol. 814, 2017, pp. 397-415. https://doi.org/10.1017/jfm.2017.34

[30] Hattori, Y., Blanco-Rodríguez, F. J., and Le Dizès, S., "Numerical stability analysis of a vortex ring with swirl," Journal of Fluid Mechanics, Vol. 878, 2019, pp. 5-36. https://doi.org/10.1017/jfm.2019.621

[31] Strutt (Lord Rayleigh), J. W., "On the dynamics of revolving fluids," Proceedings of the Royal Society of London A, Vol. 93 , No. 648, 1917, pp. 148-154. https://doi.org/10.1098/rspa.1917.0010

[32] Billant, P., and Gallaire, F., "Generalized Rayleigh criterion for non-axisymmetric centrifugal instabilities," Journal of Fluid Mechanics, Vol. 542, 2005, pp. 365-379. https://doi.org/10.1017/S0022112005006464

[33] Bayly, B. J., "Three-dimensional centrifugal-type instabilities in inviscid two-dimensional flows," The Physics of Fluids, Vol. 31, No. 1, 1988, pp. 56-64. https://doi.org/10.1063/1.867002

[34] Billant, P., and Gallaire, F., "A unified criterion for the centrifugal instabilities of vortices and swirling jets," Journal of Fluid Mechanics, Vol. 734, 2013, pp. 5-35. https://doi.org/10.1017/jfm.2013.460

[35] Meunier, P., and Leweke, T., "Three-dimensional instability during vortex merging," Physics of Fluids, Vol. 13, No. 10, 2001, pp. 2747-2750. https://doi.org/10.1063/1.1399033

[36] Lawson, N. J., and Wu, J., "Three-dimensional particle image velocimetry: experimental error analysis of a digital angular stereoscopic system," Measurement Science and Technology, Vol. 8, No. 12, 1997, pp. 1455-1464. https://doi.org/10.1088/0957$0233 / 8 / 12 / 009$ 
[37] Prasad, A. K., and Jensen, K., "Scheimpflug stereocamera for particle image velocimetry in liquid flows," Applied Optics, Vol. 34, No. 30, 1995, pp. 7092-7099. https://doi.org/10.1364/AO.34.007092.

[38] Raffel, M., Willert, C. E., Scarano, F., Kähler, C. J., Wereley, S. T., and Kompenhans, J., Particle Image Velocimetry: A Practical Guide, $3^{\text {rd }}$ ed., Springer International Publishing, Cham, 2018. https://doi.org/10.1007/978-3-319-68852-7.

[39] Jeong, J., and Hussain, F., "On the identification of a vortex," Journal of Fluid Mechanics, Vol. 285, 1995, pp. 69-94. https://doi.org/10.1017/S0022112095000462

[40] Dritschel, D. G., and Waugh, D. W., "Quantification of the inelastic interaction of unequal vortices in two-dimensional vortex dynamics," Physics of Fluids A: Fluid Dynamics, Vol. 4, No. 8, 1992, pp. 1737-1744. https://doi.org/10.1063/1.858394

[41] Brandt, L. K., and Nomura, K. K., "Characterization of the interactions of two unequal co-rotating vortices," Journal of Fluid Mechanics, Vol. 646, 2010, pp. 233-253. https://doi.org/10.1017/S0022112009992849 